\title{
Interplay between dispersive and non-dispersive modes in the polaron problem
}

\author{
L. Cruzeiro-Hansson, J.C. Eilbeck, J.L. Marín and F.M. Russell \\ Department of Mathematics, Heriot-Watt University, Edinburgh EH14 4AS, U.K.
}

(November 26, 1999)

\begin{abstract}
We study the influence of the phonon spectrum on polaron formation and show that three self-trapping regimes can occur. If the lattice and the electronlattice Hamiltonians are dominated by the same type of phonons, the selftrapping transition is smooth. If there is an imbalance, the transition can either be abrupt or completely eliminated. The binding energies are larger in the case of imbalance. The bandwidth varies linearly with hopping strength, even for strongly localized states.
\end{abstract}


The motion of quasiparticles in deformable crystals constitutes a long standing problem in solid state physics [1]. Landau was the first to suggest that a lattice distortion could induce a localized, low mobility state of the electron now known as polaron [1]. The possibility that the electron can induce a lattice deformation in which it becomes trapped was later studied in $[2-5]$ and polaron theory continues to be an active area of research [6-13]. Indeed, charge and energy localization have important consequences on properties such as conductivity, optical spectra and mechanical integrity and are thus fundamental for practical applications. An important question that remains is how to distinguish experimentally between a localized and a delocalized state. Furthermore, the possible implication of polarons or bipolarons in High Temperature Superconductivity [14-18] adds extra interest to this field.

The effect of the force range and dimensionality of the system on the self-trapping transition was investigated by Toyozawa [19] and Emin and Holstein [20]. Here we extend these studies and consider the influence of the phonon spectrum in the nature of the self-trapping transition in a one dimensional lattice. Our Hamiltonian $\hat{H}$ has three parts:

$$
\hat{H}=H_{\mathrm{ph}}+\hat{H}_{\mathrm{e}}+\hat{H}_{\mathrm{e}-\mathrm{ph}}
$$

where $H_{\mathrm{ph}}$ is the phonon Hamiltonian, $\hat{H}_{\mathrm{e}}$ is the electron Hamiltonian and $\hat{H}_{\mathrm{e}-\mathrm{ph}}$ describes the electron-phonon interaction.

The phonon Hamiltonian is:

$$
H_{\mathrm{ph}}=\frac{1}{2} \kappa_{D} \sum_{n=1}^{N}\left(u_{n}-u_{n-1}\right)^{2}+\frac{1}{2} \kappa_{E} \sum_{n=1}^{N} u_{n}^{2}+\frac{1}{2 M} \sum_{n=1}^{N} p_{n}^{2}
$$

where $u_{n}$ and $p_{n}$ are the displacement from equilibrium position and the momentum of site $n$. The first term includes dispersive, or Debye phonons, also designated as acoustic phonons in many articles; the second term includes dispersionless, or Einstein, phonons. These terms include the respective restoring constants, $\kappa_{D}$ and $\kappa_{E}$.

The electron Hamiltonian is the usual tight binding Hamiltonian:

$$
\hat{H}_{\mathrm{e}}=\epsilon \sum_{n=1}^{N} \hat{a}_{n}^{\dagger} \hat{a}_{n}-V \sum_{n=1}^{N}\left(\hat{a}_{n}^{\dagger} \hat{a}_{n-1}+\hat{a}_{n}^{\dagger} \hat{a}_{n+1}\right)
$$


where $\hat{a}_{n}^{\dagger}\left(\hat{a}_{n}\right)$ is the Fermi creation (annihilation) operator for an electron at site $n$ ( $n=$ $1, \ldots, N), \epsilon$ is the energy of the electron, and $V$ the transfer term for the electron to move between neighbouring sites.

The Hamiltonian for the electron-lattice interaction includes coupling to Debye and Einstein modes:

$$
\hat{H}_{\mathrm{e}-\mathrm{ph}}=\chi_{D} \sum_{n=1}^{N}\left(u_{n}-u_{n-1}\right) \hat{a}_{n}^{\dagger} \hat{a}_{n}+\chi_{E} \sum_{n=1}^{N} u_{n} \hat{a}_{n}^{\dagger} \hat{a}_{n}
$$

where $\chi_{D}\left(\chi_{E}\right)$ is a parameter which describes the strength of the interaction of the electron with Debye (Einstein) modes. Making $\kappa_{D}=0$ and $\chi_{D}=0$, the molecular crystal model of Holstein [4,5] is recovered. Making $\kappa_{E}=0$ and $\chi_{E}=0$, the Davydov/Scott model [21] is recovered. Mixed cases arise in many physical situations, from adsorbates on surfaces, to the Frenkel-Kontorova model of dislocations and chains of dipoles in a magnetic field. The Hamiltonian (1-4) thus covers a wide range of physical phenomena in which a quantum quasiparticle, which can be either an electron, a vacancy, or any other collective excitation, interacts with the vibrations of a lattice.

Exact calculations for the the full quantum mechanical system have been made for small systems [22-24]. For extended systems, studies in which both the electron and the lattice are treated quantum mechanically usually require approximations either regarding the relative strength of the parameters $[2,4,5]$ or in the wavefunctions considered [21,25,26,8,10-12]. Instead, we treat the electron quantum mechanically, neglect quantum effects in the lattice, and deal with this mixed quantum-classical system in an exact way over all parameter ranges [27]. The mixed quantum-classical system coincides with the adiabatic approximation in the limit of sites with infinite mass. A recent work showed good agreement between the adiabatic approximation and results obtained with the full quantum model by exact diagonalization [24]. Similar conclusions were obtained in a study which compared results of the exact mixedquantum classical system with exact results of the corresponding full quantum system [28].

Here we report on new qualitative features which, given the correspondence that can be made between the mixed quantum-classical system and the full quantum system [27], 
should also be found in the full quantum system, when treated in an exact way, i.e. avoiding approximations. Indeed, one of the main advantages of the mixed quantum-classical system is that it is trivial to show that the exact one-electron states of the mixed quantum-classical regime is:

$$
\left|\psi>=\sum_{n=1}^{N} \varphi_{n}\left(\left\{u_{n}\right\},\left\{p_{n}\right\}, t\right) \hat{a}_{n}^{\dagger}\right| 0>
$$

where $\varphi_{n}$ is the probability amplitude for an electron to be at site $n$ and its dependence on the set of displacements $\left\{u_{n}\right\}$ and momenta $\left\{p_{n}\right\}$ at time $t$ is specified by the equations of motion [27].

Averaging the Hamiltonian (1-4) over the state (5) we obtain an energy functional $\mathcal{E}\left(\left\{\varphi_{n}\right\},\left\{u_{n}\right\},\left\{p_{n}\right\}\right)$, in terms of the probability amplitudes $\left\{\varphi_{n}\right\}$ and the lattice variables $\left\{u_{n}\right\},\left\{p_{n}\right\}$. Exact minimum energy states can be found by numerically minimizing this functional with respect to all the variables, under the nonlinear condition that expresses the normalization of the wavefunction:

$$
\sum_{n=1}^{N}\left|\varphi_{n}\right|^{2}=1
$$

This minimization procedure determines the exact minimum energy states of the mixed quantum-classical system. It samples both the electron and the lattice variables at the same time and thus leads to the lattice distortion and electron probability amplitudes that correspond to the lowest energy state.

Our aim is to determine the importance of the character of the lattice modes in the minimum energy states, as a function of the parameters. In order to reduce the number of possibilities we will consider the cases in which the phonon modes have the same parameters, that is, the case in which one of them is zero, or when $\kappa_{D}=\kappa_{E}=\kappa$ and $\chi_{D}=\chi_{E}=\chi$. A non-dimensional Hamiltonian can be obtained multiplying the Hamiltonian (1-4) by $\kappa / \chi^{2}$ and substituting the displacements $\left\{u_{n}\right\}$ by the non-dimensional displacements $U_{n}=g u_{n}$, where $g=\kappa / \chi$. The non-dimensional energy functional $\mathcal{H}=\kappa / \chi^{2} \mathcal{E}$ becomes:

$$
\mathcal{H}=\frac{1}{2} \sum_{n=1}^{N}\left(U_{n}-U_{n-1}\right)^{2}+\frac{1}{2} \sum_{n=1}^{N} U_{n}^{2}+
$$




$$
\begin{aligned}
& -v \sum_{n=1}^{N}\left(\varphi_{n}^{*} \varphi_{n-1}+\varphi_{n}^{*} \varphi_{n+1}\right) \\
& +\sum_{n=1}^{N}\left(U_{n}-U_{n-1}\right)\left|\varphi_{n}\right|^{2}+\sum_{n=1}^{N} U_{n}\left|\varphi_{n}\right|^{2}
\end{aligned}
$$

where it has already been taken into account that the minimum energy states have zero site momenta $p_{n}$.

It can be seen from (6) that the first term in (3) produces only a shift in the energy equal to $\epsilon \kappa / \chi^{2}$. From equation (7) it is clear that the properties of the system in terms of the nondimensional variables $\left\{U_{n}\right\}$ and $\left\{\varphi_{n}\right\}$ can be fully characterized by the single quantity $v=V \kappa / \chi^{2}$.

We have investigated the dependence on $v$ of the minimum energy states of nine systems obtained by considering the different combinations of the two types of phonons in the lattice and in the electron-lattice interaction, as indicated in the Table. Where it says $\kappa, \chi=0$ the corresponding term in (7) has been dropped in the numerical minimization. When the different terms of the lattice and electron-lattice interaction are included, they are included with the factors they have in $(7)$.

In Fig. 1 the localization length, evaluated as $\left(\sum_{n=1}^{N}\left|\varphi_{n}\right|^{4}\right)^{-1}$, is plotted. When the state is completely localized this quantity is 1 and when it is completely delocalized, it is $N$, the number of lattice sites. In our simulations, $N=50$. We find essentially the three types of behaviour displayed in Fig. 1. For small $v$ small polarons form in all cases considered. For intermediate and large values of $v$, however, the nature of the states depends on the balance between Einstein and Debye modes. When the same type of phonons are predominant both in the lattice and in the electron-lattice interactions, as $v$ increases, intermediate size polarons arise until there is a smooth transition to a delocalized state (solid line in Fig. 1). As was shown in a previous publication [29] the threshold for delocalization increases as the number of sites $N$ increases. This smooth transition is found either when Debye, dispersive phonons dominate both the lattice and the electron-lattice interaction (the Davydov/Scott model [21]) or when Einstein, non-dispersive phonons dominate (the Holstein model $[4,5]$ ). 
Because in the Hamiltonians considered here the same weight was attributed to the Debye and Einstein oscillators and the latter overwhelm the former when they are present, this kind of smooth transition is observed, no matter what combination, as long as Einstein oscillators are present in both the lattice and the electron-lattice interactions, as the Table shows.

A second situation arises when the electron-lattice interaction includes just Debye phonons and the lattice includes Einstein oscillators (with or without Debye oscillators). Here, intermediate electron-lattice couplings do not lead to intermediate size polarons and the self-trapping transition is abrupt. Only small polarons can form at strong coupling values (dotted line in Fig. 1). The transition to a delocalized state takes place for even smaller values of $v$ when both Einstein and Debye modes are included in the lattice Hamiltonian.

A third situation results from the reverse case, in which the lattice contains essentially Debye phonons and the electron-lattice interaction contains Einstein modes (with or without Debye modes). In this case, both small and large polarons can form and the latter persist even for very weak electron-lattice couplings (see dash-dotted line in Fig. 1). In fact, the transition to delocalized states is absent in this case and at very large values of $v$, e.g. for $v>10000$, very large polarons are found, almost indistinguishable in size from delocalized states. However, the lattice displacements (not shown) are not zero, as it happens with delocalized states. A summary of the conditions under which these three situations are found is given in the Table.

Figure 2 shows the variation of the probability for an electron to be in site $n$, as a function of $v$, for the three regimes. This figure shows clearly that 1) when the phonon spectra is the same in the lattice and electron-lattice Hamiltonian, the transition from a localized to a delocalized state is smooth, 2) when the lattice is dominated by Einstein phonons and the electron-lattice interaction is dominated by Debye phonons the transition is abrupt and that 3) in the reverse case the transition is eliminated. Plots of the displacements differences $u_{n}-u_{n-1}$ are omitted because, in all cases, the extent of the lattice distortion is the same as that of the width of the corresponding probability distribution and its location is correlated 
with that of the peak of the probability distribution. The lattice displacements $u_{n}$, on the other hand have a different profile, which is extended both in thecase of Fig. 2 a and c. In the case of Fig. 2 c, a localized state is coupled to an extended lattice distortion. This is similar to what is found in $[30,31]$, in spite of the fact that in the latter the phonon spectrum is different and the electron-lattice interaction is long range, rather than local, as we considered here.

A qualitative explanation of these features can be made by inspection of the Hamiltonian (1-4). First, let us note that the electron-phonon Hamiltonian, $\hat{H}_{\mathrm{e}-\mathrm{ph}}$, is minimized by a localized electron in a localized lattice distortion. This is true both when the coupling is to Debye, dispersive phonon modes and to Einstein, dispersionless phonon modes. On the other hand, the electron Hamiltonian, $\hat{H}_{\mathrm{e}}$, is minimized by delocalized states, which arise when the displacements are zero, a condition that also minimizes the lattice Hamiltonian, $H_{\mathrm{ph}}$. Indeed, any lattice distortion leads to a positive contribution by the $H_{\mathrm{ph}}$ to the overall energy of the state. The crucial point is that this penalty is more severe for Einstein oscillators than for Debye modes. While the lattice Hamiltonian for Einstein oscillators is proportional to the displacements of each lattice site, and any non-zero displacement makes a positive contribution, the lattice Hamiltonian for Debye modes is proportional to the "space derivative" of the distortion profile. For any smooth distortion profile, the Debye term will be smaller than the Einstein term. When both the electron-lattice interaction, which induces the distortion, and the lattice Hamiltonian, which opposes the distortion, have the same type of phonons, there is a balance between the two tendencies. In this case polarons of all ranges can be found and the transition to a delocalized state is smooth. This happens when only Debye phonons are dominant, or when Einstein oscillators of equal weight are present in both the lattice and the electron-lattice interaction, and corresponds to the results displayed in the solid line of Fig. 1.

When Einstein oscillators are dominant only in the electron-lattice or only in the lattice Hamiltonian, there is an imbalance between the tendency for localization and the penalty for localization. If the lattice is dominated by Einstein phonons and the electron-lattice 
interaction is dominated by Debye phonons, for small $v$, for which the electron states are localized and the lattice distortion is sharp, the electron-phonon interaction can compensate for the penalty, and small polarons form. When $v$ increases and the electron states become less localized, leading to a broader lattice distortion, the electron-lattice interaction, which is proportional to the space derivative of the lattice distortion profile, decreases much more rapidly than the lattice energy. For intermediate values of $v$, the penalty for a localized state cannot be matched by the electron-lattice interaction and there is an abrupt transition to a delocalized state. This is as shown in the dotted line of Fig. 1. On the other hand, when the lattice is dominated by Debye phonons and the electron-lattice interaction is dominated by the Einstein phonons, the distortion induced by the latter is opposed by a weak penalty. In fact, if all the displacements are allowed to vary without bounds, the lower energy state corresponds to equal and infinite displacements which lead to $-\infty$ for the electron-lattice interaction and zero for the lattice Hamiltonian. The dash-dotted lines in figures 1-4 were obtained by setting one of the displacements equal to zero, namely $U_{N}=0$. The lattice distortion induced by the electron-lattice interaction carries only a weak penalty in the lattice energy, large polarons persist for all values of $v$, and the transition to completely delocalized states is eliminated.

A further characterization of the states is achieved by plotting their binding energies and bandwidths in Figs. 3 and 4 . The binding energy, $\mathcal{H}-2 v$, also called activation energy, is the energy needed to bring an electron to the conduction band. When the binding energy is zero, the minimum energy states are delocalized. Figure 3 shows that the small polarons that form when the lattice is dominated by Einstein oscillators and the electron-lattice by Debye oscillators have larger binding energies than those that arise in the Holstein or Davydov/Scott model (dotted line versus solid line in Fig. 3). The intermediate size polarons have comparatively low binding energies. On the other hand, the small and intermediate size polarons that form when the lattice is dominated by Debye oscillators and the electron-lattice interaction by Einstein oscillators have comparatively large binding energies (dash-dotted line in Fig. 3). 
The bandwidth plotted in Fig. 4 can be measured in an intraband Franck-Condon transition. It is the energy required to transfer an electron from the bottom to the top of the band associated with the lattice configuration of the minimum energy state. For a bare exciton, it is $4 v$. For localized, polaron states this bandwidth is larger than the bare exciton bandwidth. The small polarons that form when the lattice is dominated by Einstein oscillators and the electron-lattice by Debye oscillators have larger bandwidths than those that arise in the Holstein or Davydov/Scott model (dotted line versus solid line in Fig. 4). The intermediate size polarons have bandwidths that are very approximately those of the bare excitons, even when their size is ten times smaller. The most striking feature is shown by the small and intermediate size polarons that form when the lattice is dominated by Debye oscillators and the electron-lattice interaction by Einstein oscillators (dash-dotted line in Fig. 4). This system shows an enhancement of the bandwidth to two to three times that of the bare exciton which decreases very slowly with $v$. Also, in all cases, for $v>1$, the bandwidth varies linearly with $v$, even when the corresponding states are still localized in a few sites.

While the scaling argument in $[19,20]$ suggests that abrupt transitions and the suppression of intermediate size polarons only occur in three dimensional systems, our analysis shows that, depending on the mixing of phonons, three different types of behaviour can be found. First, smooth transitions and intermediate size polarons, second, abrupt transitions and no intermediate size polarons, and, third, only localized states, both small and large polarons. Although we have considered a one dimensional system, the argument is very general and can be applied to two and three dimensions as well. Indeed, also in higher dimensions the space derivative of a pulse is larger the sharper the pulse is, and the penalty for Einstein oscillators is higher than for Debye oscillators (see above). Furthermore, in anisotropic systems we can envisage states that are localized in one direction and delocalized in another. Finally, the argument transcends the quantum-classical divide, and while the quantitative detail may depend on the quantum behaviour of the lattice, the qualitative trends explored in this article should not. In fact, given that, for the same values of the 
parameters, the lattice distortion profile in the full quantum system is sharper than that of the mixed quantum-classical system [28], we predict that lattice quantum effects will lead to a shift of the transition to delocalized states to higher values $v$ than those reported here.

In short, we think these three regimes, which were characterized in terms of the localization length, the binding energy and the bandwidth of the electron states, categorize different situations that arise in real systems and hope that they will be of use for a systematic analysis of experimental measurements.

These studies refer to the state of electrons at equilibrium at zero or very low temperatures. They do not take into account the finite speed of lattice relaxation and cannot describe the process of localization from an initially free electron state. Also, only one electron states were considered, and electron correlations were not taken into account. These will be discussed in forthcoming papers.

We would like to thank A.M. Stoneham and A.L. Shluger for helpful discussions. J.L.M. acknowledges a Marie Curie TMR fellowship from the EU (no. ERBFMBICT972761). 


\section{REFERENCES}

[1] L. D. Landau, Phys. Z. Sowjetunion 3, 664 (1933).

[2] S. I. Pekar, Untersuchen Über die Electronentheorie der Kristalle (Akademie-Verlag, Berlin, 1954).

[3] H. Fröhlich, Proc. R. Soc. London Ser. A 160, 230 (1937).

[4] T. Holstein, Ann. Phys. 8, 325 (1959).

[5] T. Holstein, Ann. Phys. 8, 343 (1959).

[6] A. M. Stoneham, IEEE Trans. in Dielectrics and Electrical Insulation 4, 604 (1997).

[7] G. Kalosakas, S. Aubry, and G. Tsironis, Phys. Rev. B 58, 3094 (1998).

[8] A. H. Romero, D. W. Brown, and K. Lindenberg, Phys. Rev. B 60, 4618 (1999).

[9] P. Kornilovitch, Phys. Rev. B 60, 3237 (1999).

[10] T. Frank and M. Wagner, Phys. Rev. B 60, 3252 (1999).

[11] J. Bonca, S. A. Trugman, and I. Batistic, Phys. Rev. B 60, 1633 (1999).

[12] A. H. Romero, D. W. Brown, and K. Lindenberg, Phys. Rev. B 59, 13728 (1999).

[13] M. Zoli, Physica B 263, 756 (1999).

[14] A. S. Alexandrov and N. Mott, Polarons and Bipolarons (World Scientific, Singapore, Philadelphia, 1995).

[15] G.-M. Zhao, M. B. Hunt, H. Keller, and K. Müller, Nature 385, 236 (1997).

[16] A. Alexandrov, J. Superconductivity 12, 49 (1999).

[17] K. Muller, J. Superconductivity 12, 3 (1999).

[18] L. Gorkov, J. Superconductivity 12, 9 (1999). 
[19] Y. Toyozawa, Prog. Theor. Phys. 26, 29 (1961).

[20] D. Emin and T. Holstein, Phys. Rev. Lett. 36, 323 (1976).

[21] A. Scott, Physics Reports 217, 1 (1992).

[22] H. Shore and L. Sander, Phys. Rev. B 7, 4537 (1973).

[23] J. Ranninger and U. Thibblin, Phys. Rev. B 45, 7730 (1992).

[24] A. Alexandrov, V. Kabanov, and D. Ray, Phys. Rev. B 49, 9915 (1994).

[25] A. H. Romero, D. W. Brown, and K. Lindenberg, J. Chem. Phys. 109, 6540 (1998).

[26] A. Sethia, F. Hirata, Y. Tanimura, and Y.Singh, Phys. Rev. B 60, 7245 (1999).

[27] L. Cruzeiro-Hansson and S. Takeno, Phys. Rev. E 56, 894 (1997).

[28] L. Cruzeiro-Hansson and V. Kenkre, Phys. Lett. A 203, 362 (1995).

[29] L. Cruzeiro-Hansson and V. Kenkre, Phys. Lett. A 190, 59 (1994).

[30] A. Alexandrov and P. Kornilovitch, Phys. Rev. Lett. 82, 807 (1999).

[31] P. E. Kornilovitch, Phys. Rev. B 59, 13531 (1999). 


\section{TABLES}

TABLE I. Classification of self-trapping transitions.

\begin{tabular}{lccc}
\hline \hline & & $H_{\mathrm{ph}}$ & \\
\cline { 2 - 4 } & Debye & both & Einstein \\
$\hat{H}_{\mathrm{e}-\mathrm{ph}}$ & $\left(\kappa_{E}=0\right)$ & & $\left(\kappa_{D}=0\right)$ \\
\hline Debye & smooth & abrupt & abrupt \\
$\left(\chi_{E}=0\right)$ & transition & transition & transition \\
& $($ solid lines $)$ & (dotted lines) & (dotted lines) \\
both & always & smooth & smooth \\
& localized & transition & (solid lines) \\
& $($ dash-dotted $)$ & (solid lines $)$ & smooth \\
Einstein & always & smooth & transition \\
$\left(\chi_{D}=0\right)$ & tocalized & transition & (solid lines) \\
\hline \hline
\end{tabular}




\section{FIGURES}

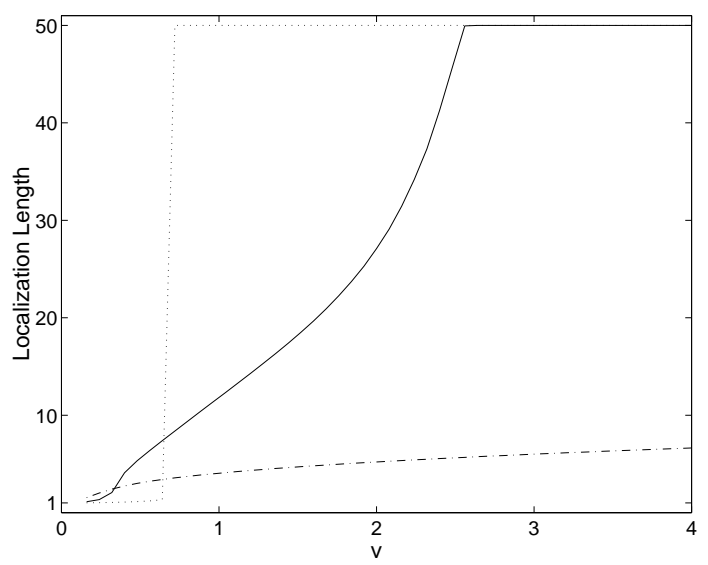

FIG. 1. Localization length (see text) versus $v$. The solid line is for $\kappa_{E}=\chi_{E}=0$, the dotted line is for $\kappa_{D}=\chi_{E}=0$ and the dash-dotted line is for $\kappa_{E}=\chi_{D}=0$.
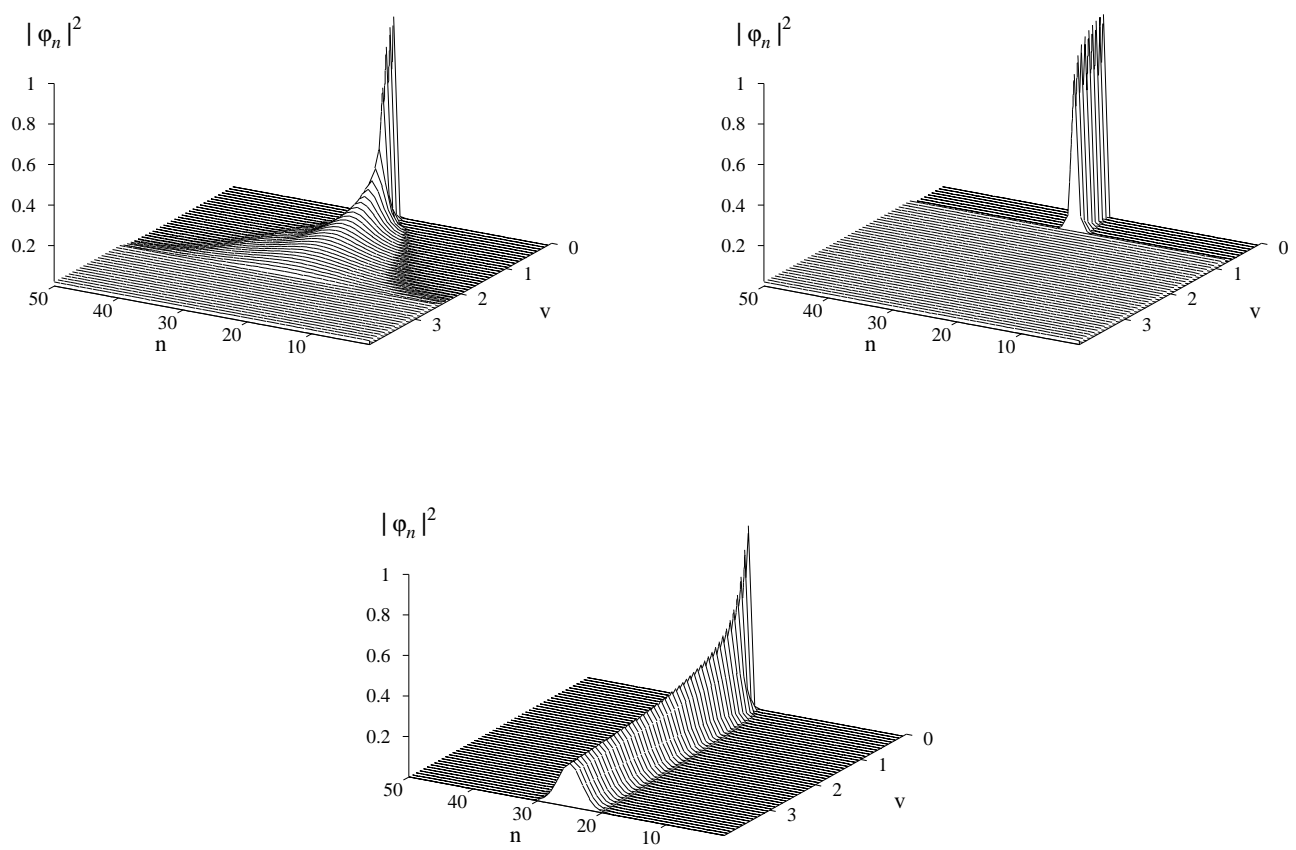

FIG. 2. Variation with $v$ (see text) of the probability for an electron in site $n$ when a) $\kappa_{E}=\chi_{E}=0$, b) $\kappa_{D}=\chi_{E}=0$ and c) $\kappa_{E}=\chi_{D}=0$. 

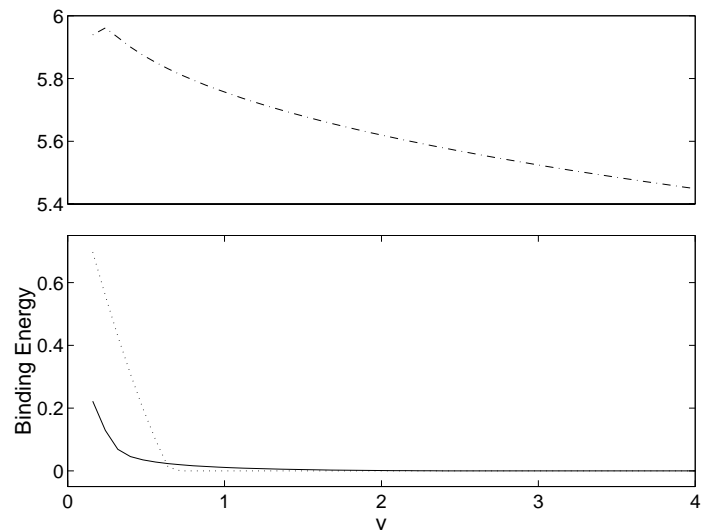

FIG. 3. Binding energy (see text) versus $v$. The line types are as in Fig. 1.

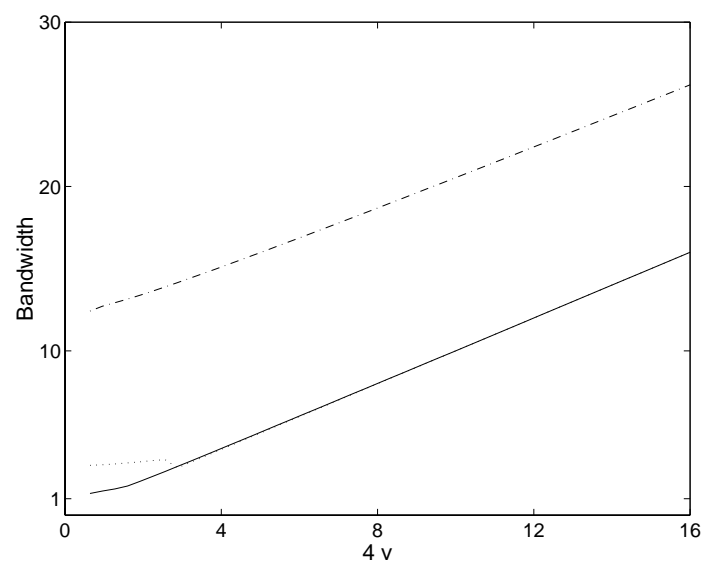

FIG. 4. Bandwidth (see text) versus $4 v$. The line types are as in Fig. 1. 